September 14, 2015

Dr. Richard Wood

Advanced Reactor Systems Group

Nuclear Science and Technology Division

Oak Ridge National Laboratory

PO Box 2008

Bldg. 3500, MS 6010

Oak Ridge, Tennessee 37831-6010

Dear Dr. Wood:

\title{
Completion of Level 4 Milestone M4AT-15OR2301039 for the Johnson Noise Thermometry for Drift-free Temperature Measurements Work Package AT-15OR230103
}

This memorandum constitutes our September 2015 level 4 milestone for the project entitled "Johnson Noise Thermometry for Drift-free Temperature Measurements" and satisfies the Milestone/Activity (Conclude HFIR field demonstration of JNT prototype). The progress summary describes the work performed to complete the subject milestone.

\section{PROGRESS SUMMARY}

During the fall of 2014 and into the winter of 2015 we placed the Johnson Noise Thermometry (JNT) system at the High Flux Isotope Reactor (HFIR) in order to subject it to a more prototypical environment than the ones previously encountered in building 3500 . The plan was to place the resistance temperature detector (RTD) in one of the process loops in HFIR. Unfortunately, replacing an existing RTD in the operating reactor proved to be untenable due to concerns about the potential impact on the safety basis and resulting extensive delays in negotiating the approval procedures. After discussions with HFIR safety management, we decided that another option existed that would allow some level of testing in a very noisy environment but would not require an overly burdensome process for deployment.

The alternate target location was the cooling tower facility behind the reactor. This location was deemed not safety critical, and we were allowed to run our RTD and cable along the edge of the water basin where there were several electrical lines going to pumps and controls for the facility. We were able to place the JNT system in a maintenance building immediately adjacent to the basin. As shown in the picture on the previous page, the building was heated and there was a wall cable entry through

Approved for public release. Distribution is unlimited. 
Dr. Richard Wood

Page 2

September 15, 2015

which we were able to easily thread the RTD and associated cable outside to the basin. There was also an Ethernet connection to the rest of the laboratory to facilitate remote monitoring.

The initial tests in August through October of 2014 consisted of the $25 \mathrm{~m}$ cable running along the periphery of the basin. We found that the motor controllers generated so much electromagnetic interference (EMI) that the amplifier system was overloaded and the measurement results were invalid. We went to a smaller length of cable (about $5 \mathrm{~m}$ ) and were able to get the error between the RTD temperature and the JNT temperature to be $0.5^{\circ} \mathrm{C}$ to $0.8^{\circ} \mathrm{C}$ even with the motors running. Occasionally we would get a run exceeding those values, and the reason was not clear. We also had a run during which the motors were not on that yielded an overnight difference of $0.16^{\circ} \mathrm{C}$, which changed to $0.13^{\circ} \mathrm{C}$ after the motors came on. This indicated that the baseline was stable even after the motors started.

The next set of tests in January 2015 were to determine whether using Cat-6 Ethernet cable connected in such a way as to provide both shielding and EMI-canceling windings would improve EMI rejection. This at first appeared to provide the desired rejection, but after further testing in a controlled, EMI-inducing coil set, it was found that this effect was not repeatable. The coil set, shown at right, was constructed to allow controlled EMI induction into JNT cables so as to be able to test the relative EMI performance of various cable configurations. Cables under test are placed horizontally between the two coils while attached to the JNT system and an RTD. The coil is then driven from a power amplifier with a square wave and the spectrum acquired. In this way, relative performance from multiple cable configurations can be obtained in a controlled environment.

In summary, the HFIR experiment showed that our in-lab testing has been a good indicator of EMI performance but that actual testing in a more prototypical environment is still needed. To this end, we will be testing at Sandia National Laboratory on a loop in October 2015.

As always, if there are questions, feel free to contact me.

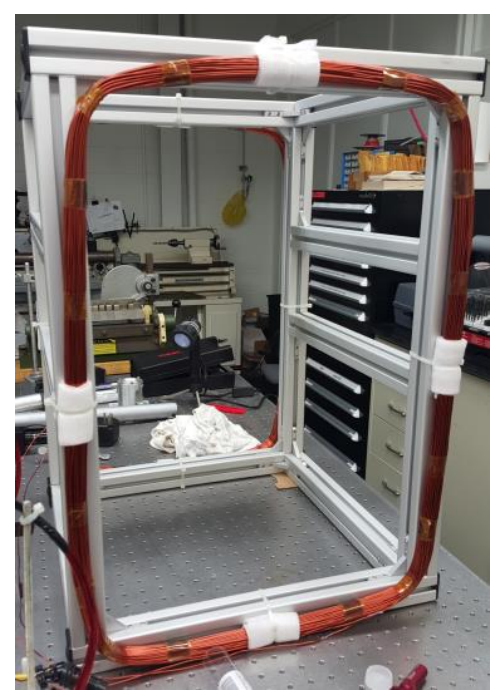

Sincerely,

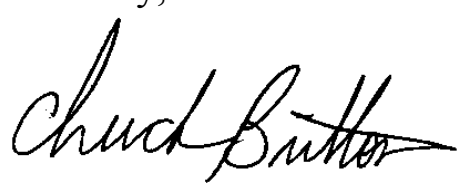

Charles L. Britton, Jr., PhD

Principal Investigator 